\title{
OSIRIS-REx spectral analysis of (101955) Bennu by multivariate statistics
}

M. A. Barucci ${ }^{1}$, P. H. Hasselmann ${ }^{1}$, A. Praet $^{1}$, M. Fulchignoni ${ }^{1}$, J. D. P. Deshapriya ${ }^{1}$, S. Fornasier ${ }^{1,2}$, F. Merlin ${ }^{1}$, B. E. Clark $^{3}$, A. A. Simon ${ }^{4}$, V. E. Hamilton ${ }^{5}$, J. P. Emery ${ }^{6}$, E. S. Howell ${ }^{7}$, J. R. Brucato ${ }^{8}$, E. A. Cloutis ${ }^{9}$, X. D. Zou ${ }^{10}$, J.-Y. Li ${ }^{10}$, P. Michel ${ }^{11}$, S. Ferrone ${ }^{3}$, G. Poggiali ${ }^{8,12}$, D. C. Reuter ${ }^{4}$, D. N. DellaGiustina ${ }^{7}$, and D. S. Lauretta ${ }^{7}$

${ }^{1}$ LESIA, Observatoire de Paris, Université PSL, CNRS, Université de Paris, Sorbonne Université, 92195 Principal Cedex Meudon, France

e-mail: antonella.barucci@obspm.fr

2 Institut Universitaire de France (IUF), Paris, France

3 Department of Physics and Astronomy, Ithaca College, Ithaca, NY 14850, USA

${ }^{4}$ NASA Goddard Space Flight Center, Greenbelt, MD, USA

5 Southwest Research Institute, Boulder, CO, USA

6 Department of Astronomy and Planetary Science, Northern Arizona University, Flagstaff, AZ, USA

7 Lunar and Planetary Laboratory, University of Arizona, Tucson, AZ, USA

8 INAF Astrophysical Observatory of Arcetri, Florence, Italy

9 Department of Geography, University of Winnipeg, Winninpeg, Canada

${ }^{10}$ Planetary Science Institute, Tucson, AZ, USA

11 Université Côte d'Azur, Observatoire de la Côte d'Azur, CNRS, Laboratoire Lagrange, Nice, France

12 University of Florence, Department of Physics and Astronomy, Firenze, Italy

Received 10 April 2020 / Accepted 1 May 2020

\section{ABSTRACT}

Contact. The NASA New Frontiers asteroid sample return mission Origins, Spectral Interpretation, Resource Identification, and Security-Regolith Explorer (OSIRIS-REx) has provided a large amount of data on the asteroid (101955) Bennu, including highquality spectra obtained by the OSIRIS-REx Visible and InfraRed Spectrometer (OVIRS).

Aims. To better constrain the surface properties and compositional variations of Bennu, we studied the visible and near-infrared spectral behavior across the asteroid surface by means of a statistical analysis aiming to distinguish spectrally distinct groups, if present.

Methods. We applied the $G$-mode multivariate statistical analysis to the near-infrared OVIRS spectra to obtain an automatic statistical clustering at different confidence levels.

Results. The statistical analysis highlights spectral variations on the surface of Bennu. Five distinct spectral groups are identified at a $2 \sigma$ confidence level. At a higher confidence level of $3 \sigma$, no grouping is observed.

Conclusions. The results at a $2 \sigma$ confidence level distinguish a dominant spectral behavior group (group 1, background) and four small groups showing spectral slope variations, associated with areas with different surface properties. The background group contains most of the analyzed data, which implies a globally homogeneous surface at the spectral and spatial resolution of the data. The small groups with redder spectra are concentrated around the equatorial ridge and are associated with morphological surface features such as specific craters and boulders. No significant variation is detected in the band area or depth of the $2.74 \mu \mathrm{m}$ band, which is associated with hydrated phyllosilicate content. The spectral slope variations are interpreted as a consequence of different regolith particle sizes, and/or porosity, and/or space weathering, that is, the presence of more or less fresh material. The OSIRIS-REx mission primary sampling site, Nightingale, and a boulder known as the Roc, are redder than the background surface.

Key words. minor planets, asteroids: individual: (101955) Bennu - methods: statistical - instrumentation: spectrographs

\section{Introduction}

The NASA asteroid sample return mission Origins, Spectral Interpretation, Resource Identification, and Security-Regolith Explorer (OSIRIS-REx) was launched on September 8, 2016, and arrived at the near-Earth asteroid (101955) Bennu on December 3, 2018. The primary objective of the mission is to collect and return to Earth a pristine sample from Bennu. For asteroid characterization and sample site selection, the OSIRIS-REx spacecraft carries two camera suites (OCAMS and
TAGCAMS), a laser altimeter (OLA), and two hyperspectral point spectrometers (OVIRS and OTES), along with a studentled X-ray imaging spectrometer (REXIS) (Lauretta et al. 2017).

The initial observations of Bennu confirmed its spinning-top shape (i.e., a spheroid with an equatorial bulge), as expected from the inversion of Earth-based radar data (Nolan et al. 2013; Lauretta et al. 2015), with a mean diameter of $490.06 \pm 0.16 \mathrm{~m}$, a sidereal rotational period of $4.296057 \pm 0.000002 \mathrm{~h}$, and a global geometric albedo of $4.4 \pm 0.2 \%$ (Lauretta et al. 2019a). Tracking the spacecraft using NASA Deep Space Network, combined 
with navigation images from the spacecraft, allowed determining the mass of Bennu and deriving a low density of $1190 \pm 13 \mathrm{~kg} \mathrm{~m}^{-3}$ (Scheeres et al. 2019; Barnouin et al. 2019).

Bennu is classified as a B-type asteroid. This type of asteroid is thought to be analogous to organic-rich, hydrated carbonaceous chondritic meteorites (Clark et al. 2011) and may have contributed to the supply of water and organic material that were essential for the emergence of life on Earth. Observations by OCAMS revealed a surface rich in craters, boulders with a wide range of sizes and unexpectedly broad albedo variations, and different regolith particle sizes (DellaGiustina et al. 2019). The boulder population varies in morphology and distribution, which may indicate variable effects of surface processes or may be linked to the formation of Bennu from the catastrophic disruption of its parent body. The analysis of the four OCAMS/MapCam filters shows spectral photometric variations associated with geologic features, including boulders, craters, and areas of mass flow (DellaGiustina et al. 2020). Moreover, a few very bright boulders of several meters were discovered by DellaGiustina et al. (2020) in the high-resolution images acquired by OCAMS; the detection of pyroxene in OVIRS spectra collected on these boulders confirmed a subordinate presence of exogenic material.

OVIRS acquired reflectance spectra from $0.4 \mu \mathrm{m}$ to $4.3 \mu \mathrm{m}$, showing blue spectra (negative spectral slopes with respect to the Sun), with a deep absorption band of hydrated phyllosilicates at $2.74 \pm 0.01 \mu \mathrm{m}$ typical of CI and CM meteorites (Hamilton et al. 2019). The analogy with these meteorites is also confirmed by OTES, which operates from 5.7 to $100 \mu \mathrm{m}$ at lower spatial resolution (Hamilton et al. 2019).

OSIRIS-REx has performed several mission phases with different observation strategies to best characterize Bennu and the prospective sampling sites. The Detailed Survey (DS) phase started with the Baseball Diamond subphase of flybys from March to April 2019, which was followed by the Equatorial Stations subphase from April to June, during which the asteroid was observed at multiple local solar times. Starting in October 2019 and extending into spring 2020, Reconnaissance (Recon A, B, and C) observations obtained the highest spatial resolution data of the candidate sampling sites identified from the DS data (Lauretta et al. 2017).

Here we use a multivariate statistical method to analyze the OVIRS spectra (level L3c) obtained at DS equatorial station 3 (EQ3). We selected EQ3 for analysis because it is the best-quality OVIRS dataset (at maximum solar illumination) that provides near-global coverage. We investigate the possible spectroscopic heterogeneity on the surface of Bennu, as we have done previously for asteroid (162173) Ryugu using data obtained by the JAXA Hayabusa2 mission (Barucci et al. 2019).

\section{Data analysis}

\subsection{OVIRS data}

The OVIRS instrument is a hyperspectral point spectrometer working in the spectral region from 0.4 to $4.3 \mu \mathrm{m}$ with a circular $4 \mathrm{mrad}$ field of view (FOV; Reuter et al. 2018, and Simon et al. 2018). During EQ3, the spacecraft was located approximately above the equator, scanning north-south at several local solar times of the day. EQ3 occurred on May 9, 2019, at local time 12:30 pm, which allowed us to obtain the best available homogeneous observations for a complete map of the surface of Bennu. The spacecraft was at a distance of $4.8 \mathrm{~km}$ from the surface of
Bennu, covering phase angles ranging from $7: 75$ to 10.37 . During this station, 17790 spots were observed with a spatial resolution per spot of about $20 \mathrm{~m}$ cross-track and $30 \mathrm{~m}$ along-track. We selected all the spectra with a fill factor $=1, i \leq 70^{\circ}$ and $e \leq 70^{\circ}$ for a total of 7151 spectra.

The data were calibrated as described by Simon et al. (2018) and corrected for illumination conditions using the shape model v20 (Barnouin et al. 2019) and the Akimov disk function model (Shkuratov 2011, Zou et al., in prep.), and the thermal tail was removed (Emery et al. 2019; Hamilton et al. 2019).

For our analysis, we filtered the spectral range from 0.5 to $3.3 \mu \mathrm{m}$ to avoid low-quality data and potential influence of thermal removal at longer wavelengths. We used as variables the spectral reflectance (normalized at $0.55 \mu \mathrm{m}$ ) at 24 wavelengths $(0.5,0.55,0.6,0.7,0.8,1,1.2,1.5,1.7,2.0,2.2,2.3,2.5,2.6$, $2.67,2.7,2.73,2.74,2.77,2.8,3.0,3.1,3.2$, and $3.3 \mu \mathrm{m})$ chosen from those that are the most significant for band detection and eliminating those with a possible overlap of order residuals after smoothing (to avoid point-to-point noise). The smoothing was performed using a Savitzky-Golay filter (Savitzky \& Golay 1964) with a polynomial of third order and a running box of five channels. A detailed analysis of the spectral region 3.0-3.6 $\mu \mathrm{m}$ is in progress (Kaplan et al. 2020).

\subsection{G-mode multivariate statistical method}

To investigate the surface compositional variation of Bennu, we applied the $G$-mode multivariate method (Gavrishin et al. 1992), which is widely used in planetary science, including recently for comet 67P/Churyumov-Gerasimenko using data obtained by the ESA Rosetta mission (Perna et al. 2017) and for Ryugu using data obtained by Hayabusa2 (Barucci et al. 2019).

The advantage of the method is that it allows the user to obtain an automatic statistical clustering of a sample containing $N$ objects (in this work, the spectra) described by $M$ variables (24 normalized reflectances at the selected set of wavelengths) in terms of homogeneous groups without any a priori criteria and taking the instrumental errors into account in measuring each variable. The original multivariate sample is collapsed into a univariate sample through successive transformations. The membership of an object in a given group (the selection criteria) is based on the statistical inference rules. The confidence level defines the probability of accepting the hypothesis that an object belongs to the given group, and this is the only a priori choice in the decision process. The objects belonging to each group follow a Gaussian distribution normalized to mean 0 and standard deviation 1 . The user selects the confidence level that corresponds to a given critical value q1: the higher $\mathrm{q} 1$, the less detailed the classification. If the user wishes to classify the whole sample with a $99.7 \%$ probability of making the correct decision in inserting a sample into a group, the critical value of $\mathrm{q} 1$ has to be 3.00 , which is the value corresponding to the $3 \sigma$ level of a standardized normal distribution (i.e., a probability of misclassifying $0.3 \%$ of the samples).

When a lower confidence level is chosen, the probability of making an incorrect decision increases, but a more detailed classification is also possible. The method also indicates the relative importance of the variables in separating the groups. We were unable to classify a few spectra because their spectral behavior is highly different from that of the identified groups. For a detailed description of the method, we refer to Barucci et al. (1987, 2019). 

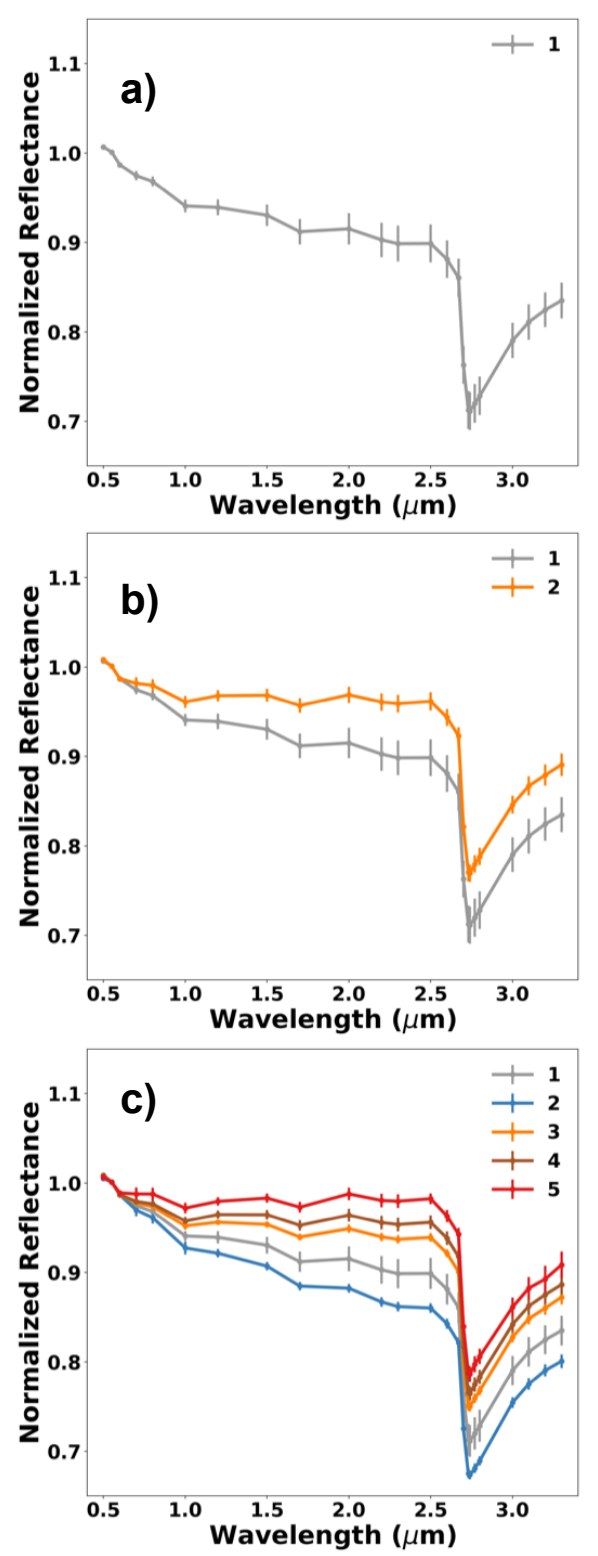

Fig. 1. Average value of variables (normalized reflectance at $0.55 \mu \mathrm{m}$ of the selected wavelengths) in groups identified from the OVIRS global data analysis: (a) average spectrum of the only groups obtained at a $3 \sigma$ confidence level, $(b)$ average spectra of the two groups distinguishable at a $2.7 \sigma$ confidence level, and $(c)$ average spectra of the five groups that can be distinguished at a $2.0 \sigma$ confidence level. The error bar corresponds to the standard deviation of the distribution of the spectra in each group and at each wavelength.

\section{Results}

We started our analysis of the 7151 spectra from EQ3 by choosing a confidence level $3 \sigma$, and we obtained only one spectral group (Fig. 1a). This implies a surface with globally homogeneous spectral properties. The spectra split into two groups only at $2.7 \sigma$ confidence level (Fig. 1b), which implies a misclassification probability of $0.7 \%$. At this confidence level, the first group (hereafter called background) contains most of the data (98\%) and has a blue mean spectrum. The smaller group contains spectra whose behavior is different from that of the background; this group represents only $2 \%$ of the data. The average spectrum of the second group is less blue (flatter slope).
A finer subdivision is apparent when the confidence level is settled at $2.0 \sigma$, which implies a misclassification probability of $4.5 \%$. The five groups at this confidence level are well separated, and the mean of each is shown in Fig. 1c. The differences among these groups are mainly due to the variation in the spectral slope; the relative histograms are shown in Fig. 2. Group 1 (background) again contains most of the spectra (89\%), an the other four groups constitute smaller fractions. The obtained groups are distinguished by their different spectral slopes mainly in the range from 1.0 to $2.5 \mu \mathrm{m}$. Group 2 (represented in blue in Fig. 1c) has a bluer slope and high reflectance. It contains about $4.5 \%$ of the total data. The other three groups (groups 3 to 5) show flatter spectral slopes (less blue) and lower mean reflectance (Figs. 2 and 3) than the background and contain 4\%, $2 \%$, and $0.5 \%$ of the total data. The reddest group (group 5), with a spectrally flat behavior, is the smallest group.

The map in Fig. 4 shows the surface distributions of the five groups. Groups 3 to 5 (with redder spectra) are distributed around the equator; at a large boulder called the Roc (centered at latitude $-24^{\circ}$, longitude $28^{\circ}$ ); in the two preselected sample sites: Nightingale (latitude $56^{\circ}$, longitude $43^{\circ}$ ) and Osprey (latitude $11^{\circ}$, longitude $88^{\circ}$ ); at a large crater of $160 \mathrm{~m}$ diameter (centered at latitude $-8^{\circ}$, longitude $269^{\circ}$ ); and in an area with higher abundance of large boulders (around latitude $-20^{\circ}$, longitude $250^{\circ}$ ), as identified by Walsh et al. (2019).

A different clustering analysis is in progress using the fourcolor-filter data obtained in the visible by MapCam (Rizos et al. 2019). The preliminary results obtained on some specific areas also shows five clusters for which the main variation is connected to albedo.

\section{Discussion and comparison with Ryugu}

The interpretation of the five identified groups is not obvious because many properties and processes overlap (particle size, porosity, space weathering, geometry effects, and temperature). Space weathering can contribute to changes in spectral slope and albedo. The red slopes might also be attributable to smaller particle size or different surface porosity. Laboratory analyses of carbonaceous chondrites (e.g., Cloutis et al. 2018) have shown a correlation between particle size and spectral slope: powdered samples with smaller particle size have redder spectra than those with larger particle sizes, and they are less compact. Stronger effects are observed in the near-infrared than in the visible wavelengths. The contribution of space weathering is not easily distinguished from particle size. No clear shift has been detected for the center of the band at $2.74 \mu \mathrm{m}$ in our groups, as expected for space weathering at these wavelengths (Lantz et al. 2015, 2017). Deshapriya et al. (2020) performed an analysis at higher spectral resolution (Recon A data) inside the Nightingale crater and detected a shift of the spectral center of band at $2.74 \mu \mathrm{m}$ toward shorter wavelengths in comparison to the mean spectrum taken out of the border of the crater. This was interpreted, following laboratory experiments (Lantz et al. 2015, 2017) on irradiated carbonaceous chondrites, as fresher material (less altered by space weathering).

The Nightingale and Osprey prime and backup sampling sites are part of groups with redder slopes. We interpret the redder spectra as indicative of smaller particle sizes and possibly of relatively fresh material. Nightingale, part of the reddest group (group 5), is known to have millimeter-scale grains from highresolution Recon imaging (Fig. 5).

A similar statistical analysis was carried out on the data of the top-shaped C-type asteroid Ryugu (Watanabe et al. 2019) 

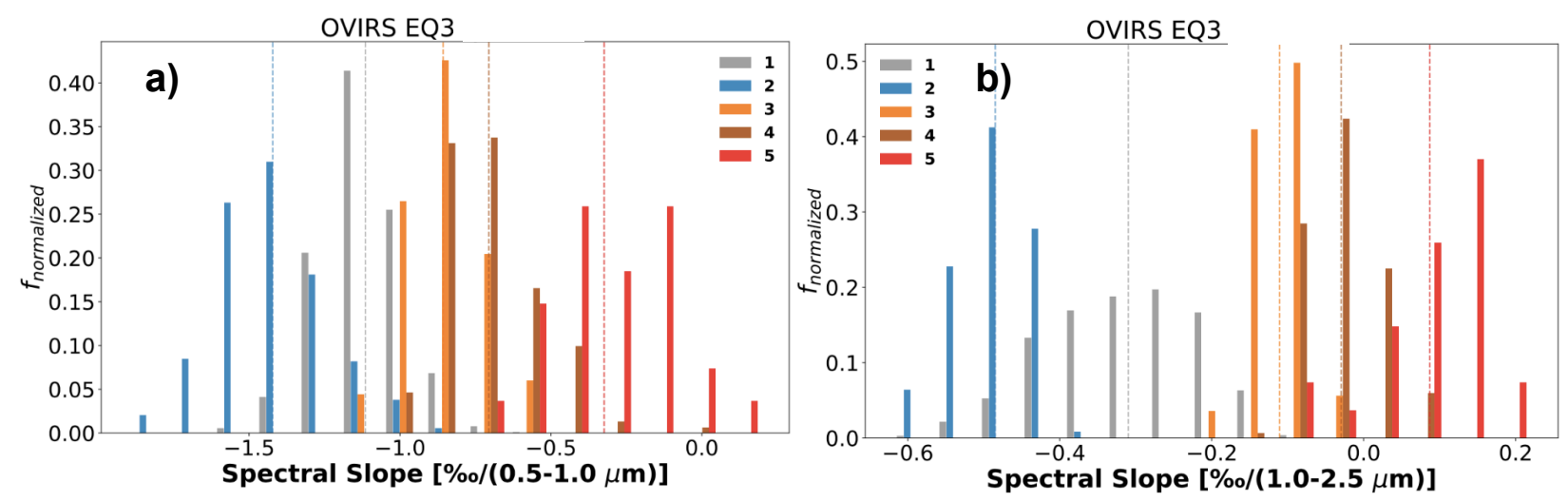

Fig. 2. Distribution of the slope of all spectra obtained for each of the five groups for $q 1=2.0 \sigma$ : $(a)$ spectral slope between 0.5 and $1.0 \mu \mathrm{m}$, and (b) spectral slope between 1 and $2.5 \mu \mathrm{m}$. The color code is the same in both panels.

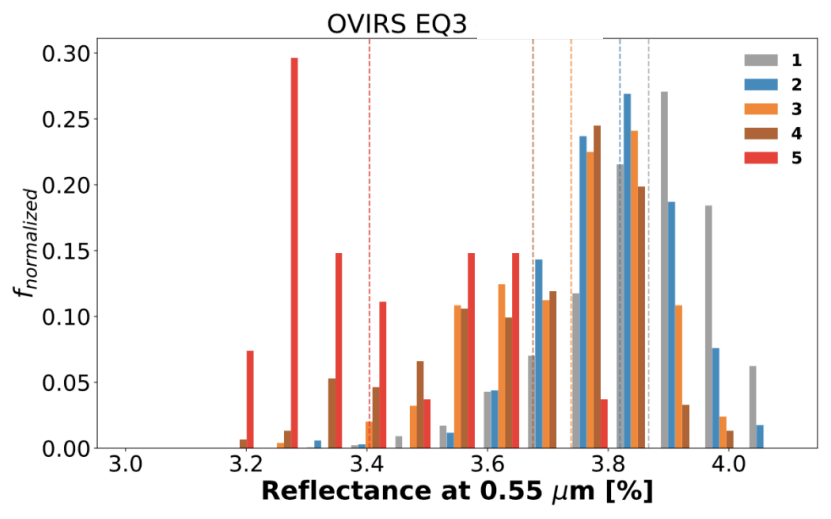

Fig. 3. Distribution of the reflectance measured at $0.55 \mu \mathrm{m}$ of the spectra for each of the five obtained groups with $q 1=2.0 \sigma$ confidence level. The reddest spectral group (group 5) has the lowest reflectance.

and obtained similar results (Barucci et al. 2019), although the spectra are different from those of Bennu. Bennu appears slightly more spectrally homogeneous than Ryugu because Ryugu showed two groups at the $3 \sigma$ confidence level. Ryugu has a red slope with a smaller hydrated band at $2.72 \mu \mathrm{m}$ associated with Mg-rich phyllosilicates (Kitazato et al. 2019). The groups detected around the equator have a bluer slope than the background. This slope has been associated with fresher material that is less strongly altered by space weathering (Sugita et al. 2019). The spectral characteristics of groups localized around the equator on Ryugu behave differently than the groups detected around the equator of Bennu, which are redder than the background. At the current spin rate of the asteroid, the motion of material on Ryugu is away from the equator (Watanabe et al. 2019; Sugita et al. 2019), which is the opposite of Bennu (Scheeres et al. 2019). The evidence on both asteroids that different spectral groups are localized on the equator might be a sign of different resurfacing directions and possibly different effects of space weathering (darkening and reddening, or brightening and blueing) that is correlated with the initial albedo and composition (Lantz et al. 2017).

\section{Conclusions}

From the results of the multivariate statistical analysis of OVIRS spectral (visible and near-infrared) data of Bennu, we confirm small spectral variations evident in the detection of statistically
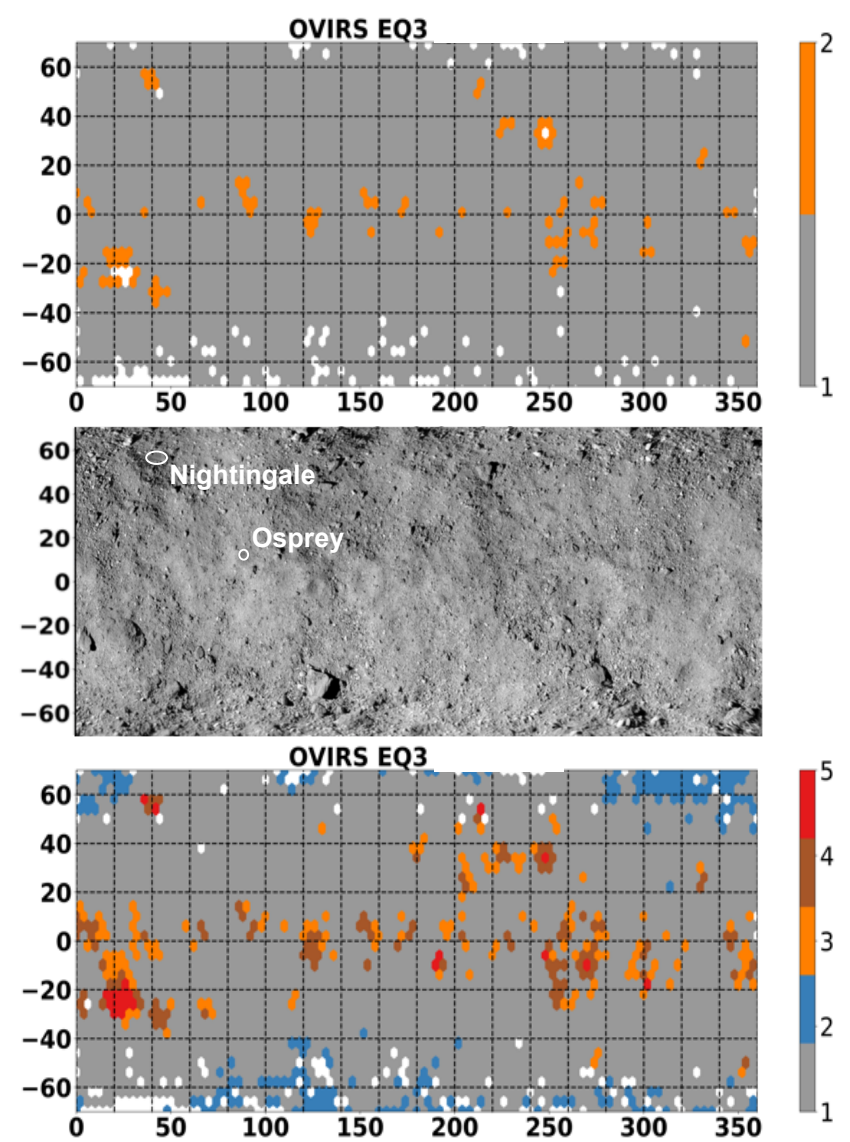

Fig. 4. Cylindrical projection maps of Bennu's surface: geographic distribution of the two groups (grey and orange) obtained at a $2.7 \sigma$ confidence level (top), map of the surface with craters and features generated from mosaicked $V$-band images as described by DellaGiustina et al. (2019) (center), and geographic distribution of the five groups obtained at $2.0 \sigma$ confidence level (bottom). White dots indicate data that are not available or not classified.

different spectral groups, whose number increases as the confidence level decreases. The statistical analysis of the spectral data at $3 \sigma$ confidence level indicates a homogeneous surface at the spectral and spatial resolution $(20 \times 30 \mathrm{~m} / \mathrm{spot})$ of the data. Slightly different areas comprising only about $2 \%$ of the analyzed data are separated into their own group at a confidence level of $2.7 \sigma$. When the confidence level is lowered to $2.0 \sigma$, four 


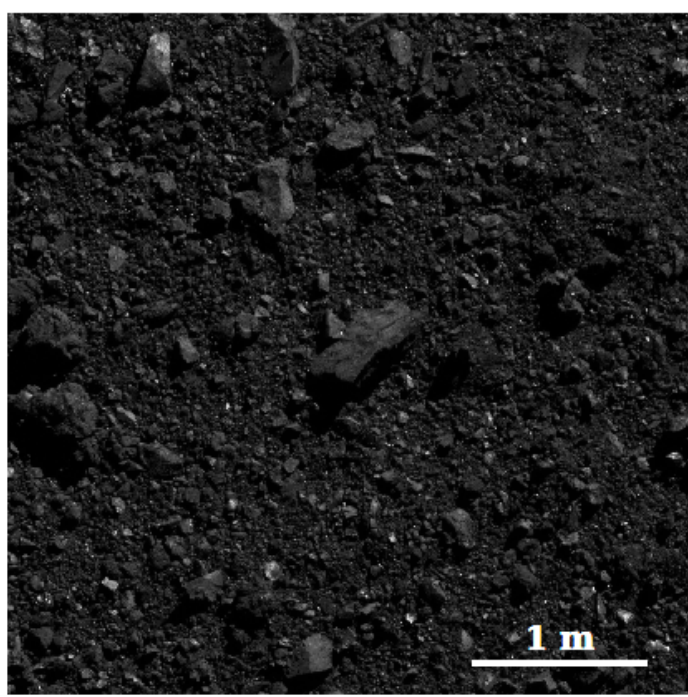

Fig. 5. Portion of the primary sample site, Nightingale (field of view, $4 \mathrm{~m}$ ), imaged by the OCAMS/PolyCam camera (Rizk et al. 2018) on March 3, 2020, from a distance of $0.3 \mathrm{~km}$. The image was obtained during the mission Recon $\mathrm{C}$ phase. The targeted touchdown point is visible in the lower left quadrant, southwest of the rock $(70 \mathrm{~cm}$ long) in the center of the image.

groups are detected that comprise about $11 \%$ of the dataset and are well separated from the predominant group, the background.

This shows that in general, Bennu has a global homogeneous surface at the resolution of the data we used, although some areas are characterized by clear variations on the spectral slopes. The identified groups show very small variation in the depth of the $2.74 \mu \mathrm{m}$ band, which implies a uniform content of hydrated phyllosilicates.

The different spectral slopes characterizing the identified statistical groups may be explained by differing particle size and porosity in the corresponding regions. Our analysis confirms that Bennu is an asteroid with a globally homogeneous surface in terms of spectral properties, although a few small regions show different degrees of variations. On the basis of our analysis, we suggest that the areas containing groups with redder (less blue) spectral slopes correspond to surface zones where the regolith is composed of particles with smaller sizes. Our work supports the sampling site selection: we confirm with the multivariate statistical analysis that the Nightingale area has the reddest spectra, implying the smallest particle sizes and possibly the freshest material. Analysis of the Recon phase observations will allow us to distinguish more variations at better spatial resolution.

These observations strongly suggest that the surface equatorial area is composed in general of material with smaller particle sizes, and/or higher porosity, and/or fresher material.

Although the spectra themselves differ, the results for Bennu and Ryugu (Barucci et al. 2019) show consistent color trends when the likely motion of material away or toward the equator is considered. Part of the equatorial regions of Ryugu, from which material moves away in the current epoch, have bluer spectra than the background, whereas the areas around the equatorial ridge of Bennu, to which material is currently directed, appear spectrally redder than the background. Bennu is slightly more spectrally homogeneous than Ryugu because a lower confidence level was needed to distinguish any groups on Bennu. The modest heterogeneity detected at this spatial resolution on the surfaces of Bennu and Ryugu is important to better understand the evolution of these two bodies. Bennu and Ryugu are rubble- pile asteroids, and the surface spectral variations are important to constrain their collisional and surface motion history.

Although different parameters (particle size, porosity changes due to accumulation or removal of material from the equator) can influence the different spectral behavior around the equator in comparison to the background spectra (redder for Bennu and bluer for Ryugu), we cannot exclude the possibility that differing effects of space weathering may be at work. This would indicate different original compositions or different thermal histories of the same composition.

Hayabusa 2 and OSIRIS-REx are expected to return pristine samples of the two asteroids to Earth in late 2020 and 2023, respectively. Laboratory analysis of the return samples will offer unique information on the nature of the two asteroids, their origin, and their evolution. Laboratory analysis of the samples will also allow direct detection of space weathering for ground truth to better interpret the surface composition from remote-sensing observations.

Acknowledgements. We are grateful to the entire OSIRIS-REx Team for making the encounter with Bennu possible. This material is based on work supported by NASA under Contract NNM10AA11C issued through the New Frontiers Program. MAB, PHH, AP, MF, JDPD, SF, FM and PM acknowledge funding support from CNES. PHH acknowledges funding support by the DIM ACAV+ program of the Region Ile de France. JRB and GP were supported by Italian Space Agency grant agreement n. 2017-37-H.0. PM acknowledges funding support from the European Union Horizon 2020 research and innovation programme under grant agreement No 870377 (project NEO-MAPP), as well as Academies of Excellence: Complex systems and Space, environment, risk, and resilience, part of the IDEX JEDI of the Université Côte d'Azur. The authors acknowledge C.W.V. Wolner for her precious support.

\section{References}

Barnouin, O. S., Daly, M. G., Palmer, E. E., et al. 2019, Nat. Geosci., 12, 247 Barucci, M. A., Capria, M., Coradini, A., et al. 1987, Icarus, 72, 304

Barucci, M. A., Hasselmann, P. H., Fulchignoni, M., et al. 2019, A\&A, 629, A13 Clark, B. E., Binzel, R. P., Howell, E. S., et al. 2011, Icarus, 216, 462 Cloutis, E. A., Pietrasz, V. B., Kiddell, C., et al. 2018, Icarus, 305, 203 DellaGiustina, D. N., Emery, J. P., Golish, R. D., et al. 2019, Nat. Astron., 3, 341 DellaGiustina, D. N., Golish, D. N., \& Burke, K. N. 2020, LPSC, 2363 Deshapriya, J. D. P., Barucci, M. A., Bierhaus, E. B., et al. 2020, Icarus, submitted

Emery, J. P., Rozitis, B., \& Christensen, P. R. 2019, EPSC-DPS2019-304-1 Gavrishin, A. I., Coradini, A., \& Cerroni, P. 1992, Earth Moon Planets, 59, 141 Hamilton, V. E., Simon, A. A., Christensen, P. R., et al. 2019, Nat. Astron., 3, 332

Kaplan, H. H., Simon, A. A., \& Emery, J. P. 2020, LPSC, 1050

Kitazato, K., Milliken, R., Iwata, T., et al. 2019, Science, 364, 272

Lauretta, D. S., Bartels, A. E., Barucci, M. A., et al. 2015, Meteorit. Planet. Sci., 50,834

Lauretta, D. S., Balram-Knutson, S. S., Beshore, E., et al. 2017, Space Sci. Rev., 212,925

Lauretta, D. S., DellaGiustina, D. N., Bennett, C. A., et al. 2019a, Nature, 568, 55

Lauretta, D. S., Antreasian, P., \& Bartels, A. E. 2019b, EPSC-DPS2019-711

Lantz, C., Brunetto, R., Barucci, M. A., et al. 2015, A\&A, 577, 41

Lantz, C., Brunetto, R., Barucci, M. A., et al. 2017, Icarus, 285, 43

Nolan, M. C., Magri, C., Howell, E. S., et al. 2013, Icarus, 226, 629

Perna, D., Fulchignoni, M., Barucci, M. A., et al. 2017, A\&A, 600, 115

Reuter, D. C., Simon, A. A., Hair, J., et al. 2018, Space Sci. Rev., 214, 54

Rizk, B., Drouet d'Aubigny, C., Golish, D., et al. 2018, Space Sci. Rev., 214, 26

Rizos, J.L., de Leon, J., \& Licandro, J. 2019, EPSC-DPS2019-169

Savitzky, A., \& Golay, M. J. E. 1964, Anal. Chem., 36, 1627

Scheeres, D. J., McMahon, J. W., French, A. S., et al. 2019, Nat. Astron., 3, 352

Shkuratov, Y., Kaydash, v., Korokhin, V., , et al. 2011, Planet Space Sci., 59, 1326

Simon, A. A., Reuter, D. C., Gorius, N., et al. 2018, Remote Sens., 10, 1486

Sugita, S., Honda, R., Morota, R., et al. 2019, Science, 364, 252

Walsh, K. J., Jawin, E. R., Ballouz, R.-L., et al. 2019, Nat. Geosci., 12, 1038

Watanabe, S., Hirabayashi, M., Hirata, N., et al. 2019, Science, 364, 268

Zou, X.-D., Li, J.-Y., Clark, B. E., et al. 2020, Icarus, submitted 